\title{
Warme Bäder gegen Depression?
}

Vielleicht sollten sich Depressive öfter mal ein heißes Bad gönnen: Eine Ganzkörperwärmebehandlung scheint jedenfalls die Stimmung deutlich zu heben - sogar über mehrere Wochen hinweg, wie US-Wissenschaftler um Dr. Clemens Janssen von der Universität in Madison in einer kontrollierten Studie zeigen konnten.Ausgangspunkt war die Beobachtung, wonach eine als angenehm empfundene Wärme bestimmte Hirnregionen wie den mittleren orbitofrontalen Kortex, das vordere Cingulum oder das ventrale Striatum aktiviert Bereiche, in denen Depressive häufig eine verminderte Aktivität aufweisen.
Die Autoren konnten 30 Patienten mit moderater Depression gewinnen. Alle unterzogen sich einer Behandlung mit einem GanzkörperhyperthermieGerät. Sie machten es sich dabei auf einer Liege bequem, während Heizspiralen und Infrarotlampen über ihnen den Körper erhitzen. In der Gruppe mit Scheintherapie $(\mathrm{n}=14)$ blieben die Infrarotstrahler aus. Nur die Heizspiralen wurden etwas aktiviert, um den Probanden das Gefühl von Wärme zu geben. Im Schnitt stieg die Körpertemperatur in der Gruppe mit aktiver Therapie um 1,9 ${ }^{\circ} \mathrm{C}$ auf $38,9^{\circ} \mathrm{C}$, in der Kontrollgruppe um $0,8^{\circ} \mathrm{C}$ auf $37,7^{\circ} \mathrm{C}$.
Schon nach einer Woche zeigten sich signifikante Unterschiede: Mit aktiver Therapie war der HAMD17-Wert um 6,1 Punkte gesunken, mit der Scheinbehandlung nur um 1,9 Punkte. Nach zwei Wochen fand sich ein Rückgang um 8,0 bzw. 4,0 Punkte, in den folgenden vier Wochen blieben die Depressions-Scores in beiden Gruppen relativ stabil. Bei den Nebenwirkungen - hauptsächlich Kopfschmerzen, Fatigue und Mundtrockenbheit - gab es keine signifikanten Differenzen.

- Janssen CW et al. JAMA Psychiatry 2016, online 12. Mai, doi: 10.1001/jamapsychiatry.2016.1031

\section{Langzeitstudie an Frauen}

\section{Bei Migräne sind Herz und Hirn in Gefahr}

Frauen mit Migräne haben nicht nur ein erhöhtes Risiko für Schlaganfälle, sondern auch für Herzinfarkte und einen vorzeitigen kardiovaskulären Tod, wie eine aktuelle Auswertung der Nurses' Health Study II ergeben hat.

Die 115.541 Teilnehmerinnen waren bei Studieneinschluss zwischen 25 und

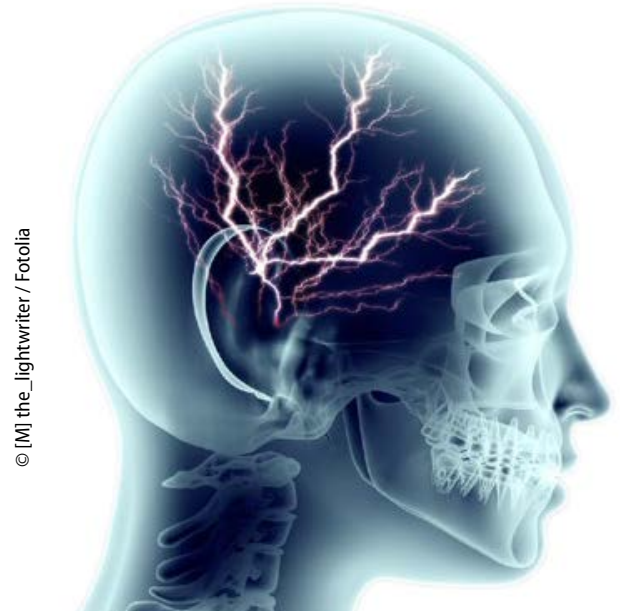

42 Jahre alt und frei von Herz-KreislaufErkrankungen. 17.531 hatten zu Beginn bereits eine Migräne, weitere $6.389 \mathrm{ka}$ men im Lauf der Studie hinzu. Während der über 20-jährigen Nachbeobachtungszeit wurden 678 Herzinfarkte, 651 Schlaganfälle und 223 Todesfälle aus kardiovaskulärer Ursache erfasst.

Nach Bereinigung um bekannte Risikofaktoren wie Alter, Hypertonie, BMI und Rauchen hatten Migränepatientinnen ein um 50\% erhöhtes Risiko für ein schweres kardiovaskuläres Ereignis, bestehend aus Schlaganfall, Herzinfarkt oder kardiovaskulär bedingtem Tod. Im Einzelnen: Herzinfarkt $+39 \%$, Schlaganfall $+62 \%$ und Angina pectoris/koronare Revaskularisation $+73 \%$ gegenüber Frauen ohne Migräne.

\section{Geschlechtsunterschiede bei Vorhofflimmern}

Hinsichtlich der Symptome und der Konsequenzen von Vorhofflimmern (VHF) gibt es deutliche Unterschiede zwischen den Geschlechtern. Dies hat ein Team der Duke University (Durham) in einer prospektiven Multicenter-Studie gezeigt. Beteiligt waren 10.135 Patienten mit VHF, darunter 4.293 Frauen. Die wichtigsten Ergebnisse:

1. Die Frauen waren im Schnitt häufiger symptomatisch und in ihren Alltagsaktivitäten beeinträchtigt und hatten eine schlechtere Lebensqualität als die männlichen Teilnehmer.

2. Weibliche Patienten erhielten häufiger als männliche eine AV-Knoten-Ablation.

3. Das Risiko, einen Schlaganfall oder eine Embolie außerhalb des ZNS zu erleiden, war bei den Frauen höher.

4. Die Gesamtüberlebensrate im Beobachtungszeitraum von im Mittel 2,3 Jahren war bei den Frauen jedoch höher und das Risiko für einen kardiovaskulären Tod geringer als bei den Männern. 